\title{
Artificial introns for effective expression of transgenes in mammalian cells
}

\author{
D.S. Naberezhnov ${ }^{1,2, *}$, E.A. Lesovaya ${ }^{2}$, K.I. Kirsanov ${ }^{2}$, M.G. Yakubovskaya ${ }^{2}$ \\ ${ }^{1}$ Engelhardt Institute of Molecular Biology, Russian Academy of Sciences, Moscow, Russia; \\ ${ }^{2}$ Federal State Budgetary Institution «N.N. Blokhin National Medical Research Center of Oncology» \\ of the Ministry of Health of the Russian Federation, Moscow, Russia; \\ *Corresponding authors: nds.xvii@gmail.ru
}

\begin{abstract}
Introns are widely used in the assembly of genetic constructions expressing transgenic proteins in eukaryotic cells for the enhancement of this expression. However, the choice of introns that can be applied for such purposes is limited by the excessively large size of the majority of natural introns (several thousand nucleotides) and therefore they cannot be cloned in a genetic construction. With the help of site-directed mutagenesis we have generated a library of short (99 nucleotides long) introns. The efficiency of these introns in the enhancement of gene expression was analyzed. As a result, a set of 12 introns was selected. The generated intros can be used for genetic constructions with high expression level of recombinant proteins.
\end{abstract}

\section{Introduction}

Introns can significantly enhance the level of gene expression. For the first time this phenomenon was found in maize [1] and later was named intron-mediated enhancement of gene expression (IME) [2]. Afterwards this effect was detected in all eukaryotes [3-5]. The mechanism of IME does not depend on the splicing efficiency and is not yet fully understood [6,7]. Some investigations show that IME can be associated with the interaction of the small nucleoprotein U1 enclosed in the structure of small nucleoprotein spliceosome with 5' splice site (5'-SS) [8-10], other splicing-involved proteins [11], histone acetylation [12], formation of DNA loops [13], mRNA export from the cell nucleus $[14,15]$, influence on translation through exon junction complex (EJC) [16].

Due to the requirement for high level of transgene expression in the molecular biological and biotechnological research, IME became widespread as a tool for expression enhancement. Small tintron, SV40 intron, rabbit $\beta$-globin intron, chimeric intron, and hybrid intron were employed for these purposes [17]. These introns represented full-size or partly truncated by excision of the internal part natural introns.

By means of rational design and site-directed mutagenesis we obtained a set of new artificial introns not existing in nature, but containing elements universal for all introns. 
Elements essential for intron splicing differ depending on intron type; for introns of protein coding genes the essential elements are represented by donor and acceptor splice sites 5'-SS and 3'-SS, branching point, and polypyrimidine sequence [18,19] (Fig. 1).

In the 5'-SS of intron the sequence of nucleoprotein U1 RNA binding is localized [20], 3'-SS sequence and polypyrimidine sequence contain sequences of small (35 kDa) subunit U2AF binding [21] and polypyrimidine tract-binding protein (PTB) responsible for splicing regulation binding [22] respectively, and the branching point contains the sequence of large (65 kDa) subunit U2AF binding [23]. Artificial introns must contain all these elements for effective splicing.

Natural introns are characterized by wide distribution of lengths from 50 to several thousand nucleotides with the pick value of 100-200 nucleotides [24] and diverse splicing efficiency correlated with the length and IME [25,26]: longer introns demonstrate better efficiency. For molecular biological investigations short introns are preferable, therefore we have chosen the length of 99 nucleotides for the generation of artificial introns.

\section{Materials and methods}

\section{Molecular cloning}

Plasmids were obtained by standard restriction - ligation method and Golden Gate method. PCR conditions, sequences of plasmids and primers are presented in the Supplement.

The library of plasmids pSB/IR-CA-HybIntr-2x99intr-SV40intr-BleoR-T2A-mCherry was obtained by cloning of the library of DNA duplexes generated by renaturation of oligonucleotides 13099intr-F and 131-99intr-R (with completion of complementary DNA T4 strands with DNApolymerase and restriction by endonucleases BsaI (NEB, USA) and KpnI) into pSB/IR-CA-HybIntrSV40intr-BleoR-T2A-mCherry plasmid restricted with restriction endonucleases BsaI (NEB, USA) and AsiG.

Cell line culturing. HEK293T cell line was cultivated in 24-well plates in DMEM medium (Paneco, Russia) with $10 \%$ calf embryonic serum (Paneco, Russia) in the incubator with $5 \% \mathrm{CO}_{2}$ at $37^{\circ} \mathrm{C}$.

Transfection. 5 hours before transfection the cell culture medium was changed. The cells were transfected with the help of TurboFect (Thermo Fisher Scientific, USA) according to the manufacturer's instructions. Plasmid DNA for transfection was isolated using Plasmid Miniprep Kit (Evrogen, Russia) according to the manufacturer's instructions. In 2 days the medium was changed, and a new portion with $100 \mathrm{mcg} / \mathrm{ml}$ antibiotic zeocin was added.

Fluorometry. Fluorometry was performed on Fluoroskan FL (Thermo Fisher Scientific, USA) with FL1 filters. 


\section{Results}

Artificial introns (99 intron $\mathrm{N}$, where $\mathrm{N}$ is the serial number of intron) were generated by sitedirected mutagenesis with subsequent selection of variants capable of undergoing splicing. For this purpose, we designed a plasmid pSB/IR-CA-HybIntr-SV40intr-BleoR-T2A-mCherry in which under the control of promoter CAG the gene of zeocin resistance fused through T2A peptide with mCherry protein was placed (Fig. 2). Such plasmid construction is designed so that the artificial intron insertion splits the reading frame of zeocin resistance gene, and if the intron is not capable of splicing the resistance gene remains inactive. This plasmid construction allows selection of intron variants capable of splicing with the help of antibiotic zeocin.

The general intron design was developed based on acknowledged literature data. GTAAGT sequence complementary to the sequence of spliceosome RNA U1 was chosen as 5'-SS intron sequence [27]. CAG sequence common for all introns was used as 3'-SS, and TACTAAC sequence complementary to the sequence of spliceosome RNA U2 served as the branching point sequence. The distance from branching sequence to 3'-SS and the length of polypyrimidine sequence were also chosen as the most frequently found in introns [27]. Restrictase BbsI sequence was also enclosed into intron for further modifications.

The sequence TAAGT(N) ${ }_{12}$ YCTAGYNGTCTTC $(\mathrm{N})_{39}$ TACTAAC $(\mathrm{N})_{4}(\mathrm{Y})_{15} \mathrm{CAG}$ represents the common sequence of artificial intros. Introns 99 intron $\mathrm{N}$ were combined in dimers (in order to increase the number of intron variants) and the obtained library was cloned into plasmid pSB/IR-CAHybIntr-SV40intr-BleoR-T2A-mCherry. The generated plasmid library was transfected into HEK293T cell line with subsequent selection with zeocin and choice of clones resistant to zeocin and hence containing introns capable of splicing.

From the clones correctly expressing zeocin resistance protein 250 intron variants were extracted for further verification of splicing efficiency and capability to enhance gene expression. The generated introns were sequenced. And as a result of this sequencing it was found that only 12 introns had full-size 99 nucleotide length, and the rest were 48 nucleotides long, but contained 5'-SS and 3'SS sequences, and polypyrimidine tract. In some introns the branching point sequence TACTAAC was absent.

For the verification of the characteristics of 12 full size introns they were recloned into plasmid pSB-IR-pA-Pause_Site-CAG-TurboGFP-MODC-IntrHBB-bGHpA-SV40pr-BleoR-SV40pA

containing fluorescent protein TurboGFP gene (Fig 3b), so that TurboGFP was capable of fluorescence only if correct intron splicing occurred. Capability of the enhancement of protein expression was checked by means of fluorometry; plasmid variants without intron and with SV40 
intron were used as controls. Fluorescence levels are shown at Figure 3a. All generated introns were capable to enhance the efficiency of expression 8-12 fold that is comparable with expression enhancement with SV40 intron. Differences in the enhancement induced by individual introns were practically unobserved.

Thus, we have generated a set of introns possessing high splicing efficiency and capable of effective gene expression enhancement.

\section{Competing interests}

The authors declare no competing interests.

\section{Acknowledgement}

The study was supported by RFBR in the frames of scientific project 19-34-60031.

\section{References}

1. Callis J, Fromm M, Walbot V (1987) Introns increase gene expression in cultured maize cells. Genes \& Development 1: 1183-1200.

2. Mascarenhas D, Mettler IJ, Pierce DA, Lowe HW (1990) Intron-mediated enhancement of heterologous gene expression in maize. Plant Mol Biol 15: 913-920.

3. Chung S, Perry RP (1989) Importance of introns for expression of mouse ribosomal protein gene rpL32. Molecular and Cellular Biology 9: 2075-2082.

4. Meredith J, Storti RV (1993) Developmental regulation of the Drosophila tropomyosin II gene in different muscles is controlled by muscle-type-specific intron enhancer elements and distal and proximal promoter control elements. Dev Biol 159: 500-512.

5. Walton SJ, Wang H, Quintero-Cadena P, Bateman A, Sternberg PW (2020) Caenorhabditis elegans AF4/FMR2 Family Homolog affl-2 Regulates Heat-Shock-Induced Gene Expression. Genetics 215: 1039-1054.

6. Shaul O (2017) How introns enhance gene expression. Int J Biochem Cell Biol 91: 145-155.

7. Akua T, Berezin I, Shaul O (2010) The leader intron of AtMHX can elicit, in the absence of splicing, low-level intron-mediated enhancement that depends on the internal intron sequence. 10: 93.

8. Kwek KY, Murphy S, Furger A, Thomas B, O'Gorman W, et al. (2002) U1 snRNA associates with TFIIH and regulates transcriptional initiation. Nat Struct Biol 9: 800-805.

9. Damgaard CK, Kahns S, Lykke-Andersen S, Nielsen AL, Jensen TH, et al. (2008) A 5? Splice Site Enhances the Recruitment of Basal Transcription Initiation Factors In Vivo. Molecular Cell 29: 271-278.

10. Kaida D, Berg MG, Younis I, Kasim M, Singh LN, et al. (2010) U1 snRNP protects pre-mRNAs from premature cleavage and polyadenylation. Nature 468: 664-668.

11. Lin S, Coutinho-Mansfield G, Wang D, Pandit S, Fu XD (2008) The splicing factor SC35 has an active role in transcriptional elongation. Nat Struct Mol Biol 15: 819-826.

12. Nicole, Fernando, Straube K, Karla (2012) First Exon Length Controls Active Chromatin Signatures and Transcription. 2: 62-68.

13. Moabbi AM, Agarwal N, El Kaderi B, Ansari A (2012) Role for gene looping in intron-mediated enhancement of transcription. Proceedings of the National Academy of Sciences 109: 8505-8510.

14. Dimaano C, Ullman KS (2004) Nucleocytoplasmic Transport: Integrating mRNA Production and Turnover with Export through the Nuclear Pore. Molecular and Cellular Biology 24: 3069-3076.

15. Valencia P, Dias AP, Reed R (2008) Splicing promotes rapid and efficient mRNA export in mammalian cells. Proc Natl Acad Sci U S A 105: 3386-3391.

16. Lee HC, Choe J, Chi SG, Kim YK (2009) Exon junction complex enhances translation of spliced mRNAs at multiple steps. Biochem Biophys Res Commun 384: 334-340. 
17. Gray SJ, Foti SB, Schwartz JW, Bachaboina L, Taylor-Blake B, et al. (2011) Optimizing Promoters for Recombinant Adeno-Associated Virus-Mediated Gene Expression in the Peripheral and Central Nervous System Using Self-Complementary Vectors. Human Gene Therapy 22: 1143-1153.

18. Shi Y (2017) Mechanistic insights into precursor messenger RNA splicing by the spliceosome. Nat Rev Mol Cell Biol 18: 655-670.

19. Krämer A (1996) The structure and function of proteins involved in mammalian pre-mRNA splicing. Annu Rev Biochem 65: 367-409.

20. Lerner MR, Boyle JA, Mount SM, Wolin SL, Steitz JA (1980) Are snRNPs involved in splicing? Nature 283: 220-224.

21. Wu S, Romfo CM, Nilsen TW, Green MR (1999) Functional recognition of the 3' splice site AG by the splicing factor U2AF35. Nature 402: 832-835.

22. Wagner EJ, Garcia-Blanco MA (2001) Polypyrimidine Tract Binding Protein Antagonizes Exon Definition. Molecular and Cellular Biology 21: 3281-3288.

23. Graveley BR, Hertel KJ, Maniatis T (2001) The role of U2AF35 and U2AF65 in enhancer-dependent splicing. 7: 806-818.

24. Sakharkar MK, Perumal BS, Sakharkar KR, Kangueane P (2005) An analysis on gene architecture in human and mouse genomes. In Silico Biol 5: 347-365.

25. Bradnam KR, Korf I (2008) Longer First Introns Are a General Property of Eukaryotic Gene Structure. PLoS ONE 3: e3093.

26. Li SW, Feng L, Niu DK (2007) Selection for the miniaturization of highly expressed genes. Biochem Biophys Res Commun 360: 586-592.

27. Wilkinson ME, Charenton C, Nagai K (2020) RNA Splicing by the Spliceosome. Annu Rev Biochem 89: 359388. 


\section{Branch point}

\section{Exon $5^{\prime}$-SS Poly $(\mathrm{Y})$ tract $=3^{\prime}$-SS Exon}

Figue 1. General scheme of intron.

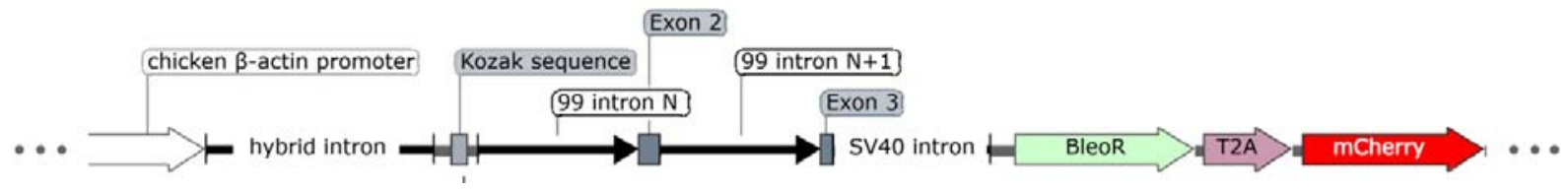

Figure 2. Scheme of expression cassette applied for the selection of artificial introns.

a

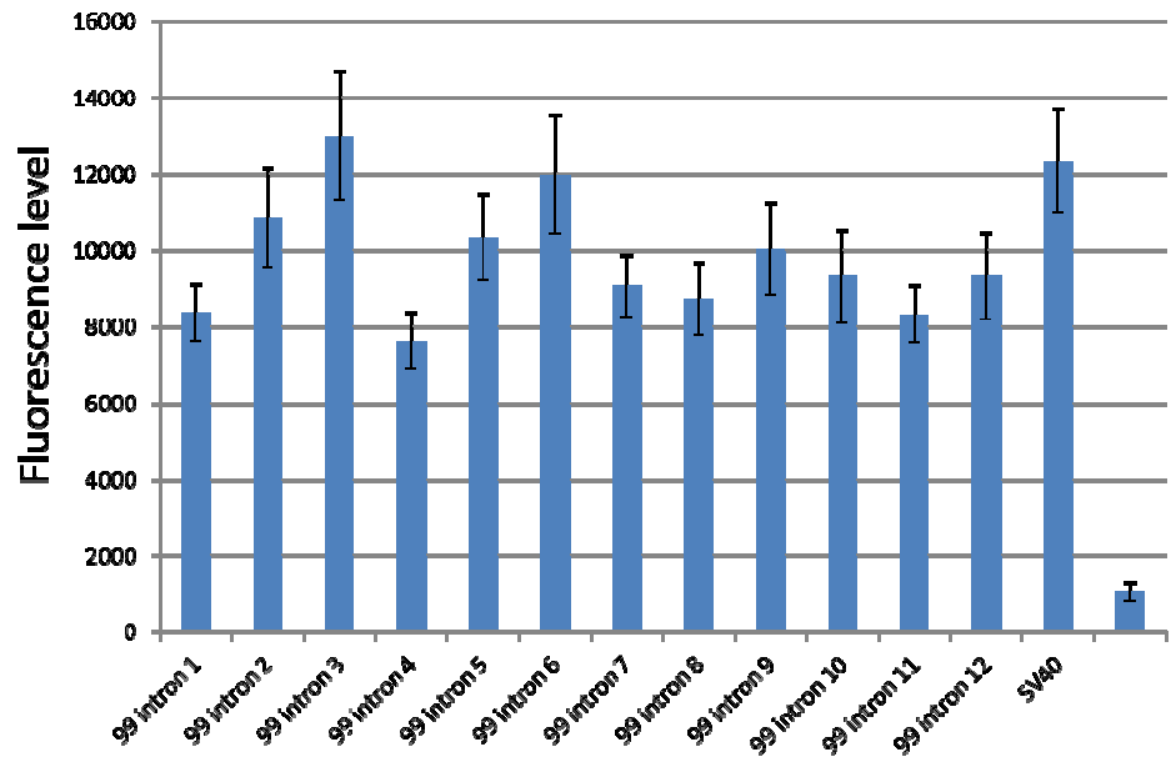

b

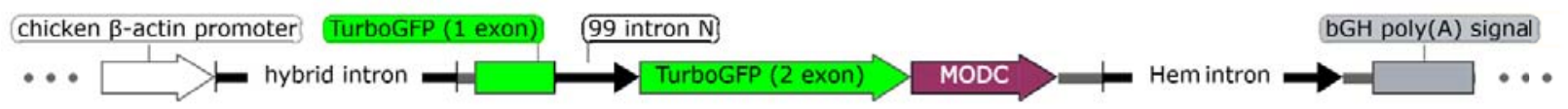

Figure 3. Fluorescence measurement of TurboGFP protein containing the studied introns (a); scheme of the cassette applied for expression investigations (b). 